\title{
Modeling and analysis of regenerative cooling channels for scramjet engine
}

\author{
Feng Gao, Qian Zhanga , Hongyu Xiao, Chengtao Zhang, Xuefeng Xia \\ Air and Missile Defense College, Air Force Engineering University, 710051 Shaanxi Xian, China
}

\begin{abstract}
This paper presents a CFD simulation analysis method for the regenerative cooling channel of a supercombustion ramjet engine, and performs three-dimensional modeling and analysis. The influences of inlet mass flow, inlet pressure, and inlet temperature on the flow and heat transfer characteristics of kerosene in the cooling channel were analyzed, and the conclusions are as follows: The larger the inlet mass flow, the lower the maximum wall temperature and oil temperature. The change trend of wall temperature is basically the same under different inlet pressure conditions, all of which increase violently first, then stabilize and then suddenly increase suddenly, then decrease almost to the outlet, and the temperature is almost the same under different conditions. As the inlet temperature decreases, the temperature difference between the wall surface and kerosene becomes larger, and the convective heat transfer coefficient gradually increases.
\end{abstract}

\section{Introduction}

With the increasing number of flying Mach numbers, the thermal protection problem of hypersonic vehicles becomes more and more serious. The work of supercombustion stamping engines is in extreme harsh environments such as high speed, high heat flux density, high-intensity combustion, etc., and a feasible and reasonable thermal protection is urgently needed. Due to the particularity of the working environment of the combustion chamber, the commonly used combustion chamber cooling methods include film cooling, ablation cooling, perspiration cooling, and regenerative cooling ${ }^{[1]}$. Compared with other types of cooling methods, regenerative cooling has great advantages. At present, the more successful hypersonic aircrafts in the world such as X-43A, X-51A and other super-combustion stamping engines use regenerative cooling technology. A key technology in supersonic vehicle technology. In recent years, with the continuous maturity of computer technology, numerical simulation has also been widely used, and the research on regenerative cooling technology at home and abroad has also achieved considerable achievements [2-10]. However, most scholars have simplified the cooling channel based on the criterion relationship or based on some flow hypothesis to build a simple one-dimensional heat transfer model. There are relatively few numerical simulations of three-dimensional channels, and most of them have studied the heat transfer characteristics of the coolant in the channels, and there is less research on the effect of the inlet conditions of the coolant on the cooling efficiency. The channel was modeled by 3D CFD, and calculated and analyzed. The flow and heat transfer characteristics of kerosene in the channel under different inlet mass flow, inlet pressure, and inlet temperature are analyzed.

\section{Calculation models and numerical methods}

\subsection{Geometri cmodel and basic assumptions}

A super-combustion stamping engine using a regenerative cooling channel is welded from different heat exchange panels. The simple structure is shown in Figure 1. The schematic diagram of the heat exchange plate with regenerative cooling channel is shown in Figure 2.

a Corresponding author: zhangqian8913@163.com 


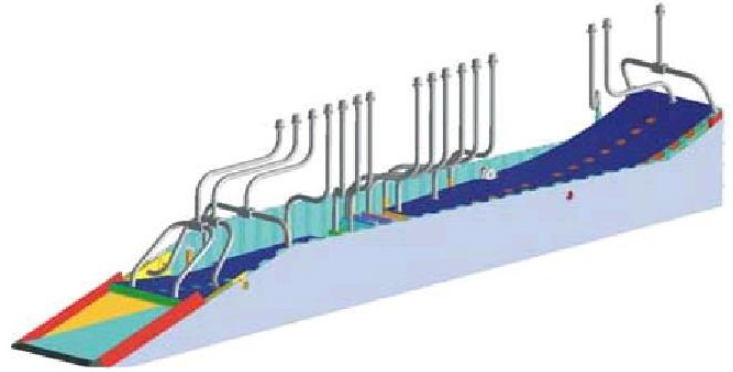

Figure 1. Scramjet engine with regenerative cooling.

Due to the symmetry of the regenerative cooling channel, in order to simplify the calculation, only one half of the flow unit of the cooling channel is analyzed. Figure 3 is a cross-sectional view of the half of the flow unit of the regenerative active cooling channel. Where $\mathrm{a}$ is half the rib width, $b$ is the height of the cooling channel, $\mathrm{c}$ is the half width of the channel, $d$ is the thickness of the outer wall, and $\mathrm{e}$ is the thickness of the inner wall. The channel material is made of alloy steel, and the coolant is made of domestic aviation kerosene. Its physical properties are given in ${ }^{[11]}$.

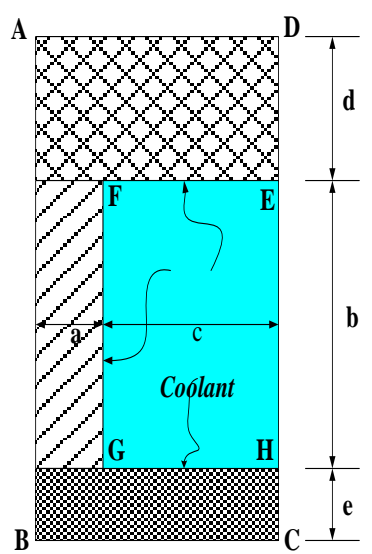

Figure 3. Sectional view of flow unit of regenerative active cooling channel flow unit.

Due to the complexity of heat transfer in regenerative active cooling channels, the following assumptions are

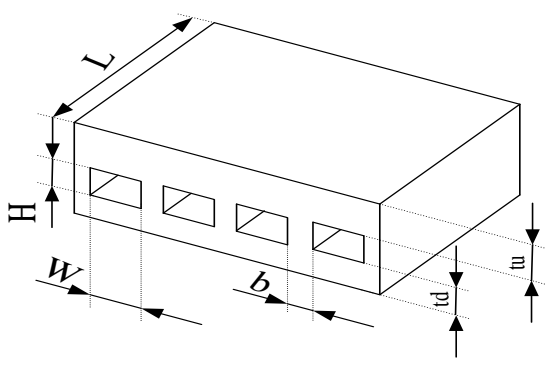

Figure 2. Heat exchange plate for regenerative cooling channel.

made on the theoretical model:

(1) The fluid in the channel has no phase change latent heat release and absorption. The heat transfer process has the characteristics of unidirectional flow, and the fluid is in a supercritical state in the channel;

(2) The cross-sectional shape of the channel is regarded as a rectangle;

(3) The thermal resistance between the connections is negligible;

(4) The symmetry plane of the side ribs is an adiabatic surface;

(5) Do not consider the influence of radiative heat transfer;

(6) Dissipate heat from the channel to the environment;

(7) Do not consider the carbon deposits of aviation kerosene.

\subsection{Numerical calculation method}

\subsubsection{Governing Equation}

The governing equations include mass continuity equations, energy equations, and momentum equations, as shown in equations (1), (2) and (3), respectively.

$$
\frac{\partial \rho}{\partial t}+\frac{\partial}{\partial x_{i}}\left(\rho u_{i}\right)=0
$$

In the formula (2), $k_{\text {eff }}$ is the effective thermal conductivity: $k_{\text {eff }}=k_{l}+k_{t}, k_{t}$ is the turbulent thermal conductivity, which is determined based on the turbulence model. ${ }^{J}{ }_{j}$ is the diffusion flux of component $j^{\prime}, S_{h}$ contains the heat of chemical reaction and other user- 
defined volumetric heat source terms, $E=h-\frac{p}{\rho}+\frac{u_{i}^{2}}{2}$

In equation (3), $\rho g_{i}$ and $F_{i}$ are the gravitational volume force and the external volume force in the $\mathrm{i}$

direction, respectively, and $\tau_{i j}$ is the stress tensor:

$\tau_{i j}=\left[\mu\left(\frac{\partial u_{i}}{\partial x_{j}}+\frac{\partial u_{j}}{\partial x_{i}}\right)\right]-\frac{2}{3} \mu \frac{\partial u_{l}}{\partial x_{l}} \delta_{i j}$

In the formula,

$\mu_{\text {is the viscosity coefficient: }} \mu=\mu_{l}+\mu_{t}, \mu_{l}$ is the

laminar viscosity coefficient, $\mu_{t}$ is the turbulent

viscosity coefficient, which is defined according to the

turbulence model.

\subsubsection{Meshing and boundary conditions}

Simulation conditions: The mass flow rate of the coolant aviation kerosene inlet is $0.01 \mathrm{~kg} / \mathrm{s}$, the calculated area is length $0.5 \mathrm{~m}$, width $1.5 \mathrm{~mm}$, heat flow density of the inner wall $1 \mathrm{MW} / \mathrm{m}^{2}$, and inlet pressure $4 \mathrm{MPa}$. Channel parameters: The thickness of the half rib is $1 \mathrm{~mm}$, the channel is $4 \mathrm{~mm}$ high, $4 \mathrm{~mm}$ wide, the outer wall thickness is $3 \mathrm{~mm}$, and the inner wall thickness is $2 \mathrm{~mm}$.

In order to simplify the problem, the horizontal heat transfer between adjacent channels is not considered, and the symmetry is used to reduce the number of meshes and shorten the calculation time. The fluid-solid coupling interface is encrypted when the mesh is divided, compared with the number of 200,000 meshes and 600,000 meshes. As a result of the calculation, the temperature difference is within the range of $15 \mathrm{~K}$, so the calculation uses 200,000 grids. The grid division is shown in Figure 4.

In numerical calculations, proper and correct boundary conditions are of great significance to the accuracy of the calculation. Different boundary conditions are chosen to obtain different results.

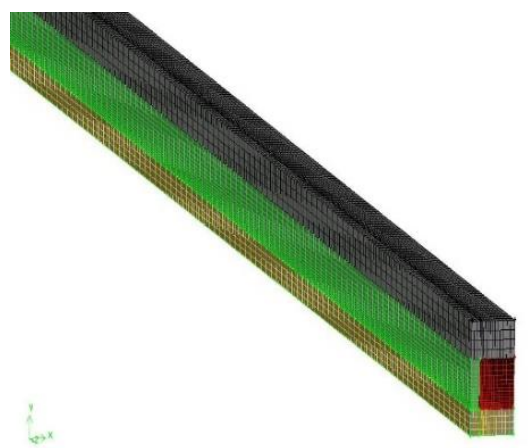

Figure 4. Cooling channel meshing.
The inlet AO is the boundary condition for the

mass flow into the inlet, mass flow $\dot{m}=\dot{m}_{0}$, inlet pressure $p=p_{0}$, inlet temperature $T=T_{0}$, turbulent kinetic energy $k=k_{0}$, and turbulent dissipation rate $\varepsilon=\varepsilon_{0}$, where $k_{0}$ and $\varepsilon_{0}$ are shown by equations (4) and (5) respectively.

$$
\begin{gathered}
k_{0}=\frac{3}{2}\left[\bar{u}\left(0.16 R e_{D H}\right)^{-1 / 8}\right]^{2} \\
\varepsilon_{0}=C_{u}^{3 / 4} \frac{k^{3 / 2}}{l}
\end{gathered}
$$

Where $\bar{u}$ is the average fluid velocity, $\mathrm{DH}$ is the hydraulic diameter, $C_{u}$ is the empirical constant, 0.09, and $l$ is the turbulence length scale, which is approximately $0.07 \mathrm{D}$.

The upper wall surface, the outlet wall and the inlet wall surface are adiabatic wall surfaces, and the wall surface heat flow density is zero. The lower wall surface is the wall surface of the heating section, and the heat flux density is the heat flux density applied in the physical model. Both sides are set to symmetrical boundary conditions, and the axial velocity, temperature, and pressure are all zero. The outlet cross section is a pressure outlet, and the outlet back pressure is set to zero.

\subsubsection{Solving the governing equations}

The governing equation is solved according to incompressible and variable physical conditions. Fluent was used for numerical simulation. The RNG $k-\varepsilon$ model was used for the turbulence model. The equation was discretized using the second-order upwind style. The SIMPLEC algorithm was used to solve the coupling of pressure and velocity. All variables in the governing equation have second-order accuracy in time and space.

\section{Calculation results and analysis}

\subsection{Impact of inlet mass flow}

In order to analyze the effect of different mass flow on the heat transfer performance of the cooling channel, this section only changes the kerosene inlet mass flow without changing the channel geometry, inlet temperature and inlet pressure, and simulates the situation of 10 different mass flow inlets. The parameters and results are as follows: 
Table 1. Comparison of calculation results of different inlet mass flows

\begin{tabular}{c|c|c|c|c|c}
\hline Study No. & $\begin{array}{c}\text { Quality flow } \\
(\mathrm{g} / \mathrm{s})\end{array}$ & $\begin{array}{c}\text { Inlet temperature } \\
(\mathrm{K})\end{array}$ & $\begin{array}{c}\text { Inlet pressure } \\
(\mathrm{MPa})\end{array}$ & $\begin{array}{c}\text { Maximum wall temperature } \\
(\mathrm{K})\end{array}$ & $\begin{array}{c}\text { Maximum oil temperature } \\
(\mathrm{K})\end{array}$ \\
\hline 1 & 4 & & 1254.927 & 1010.276 \\
2 & 6 & & 1062.356 & 900.240 \\
3 & 8 & & 905.512 & 813.661 \\
4 & 10 & 300 & 4 & 825.071 & 748.982 \\
5 & 15 & & 810.710 & 699.982 \\
6 & 20 & & 801.235 & 680.021 \\
7 & 25 & & 792.001 & 672.104 \\
8 & 30 & & & 770.842 & 653.943 \\
9 & 40 & & & 762.570 & 640.009 \\
10 & 50 & & & 738.993 & 633.182 \\
\hline
\end{tabular}

It can be seen from Table 1 that as the inlet mass flow rate increases, the maximum wall temperature and maximum oil temperature are decreasing. In order to more clearly analyze the relationship between the inlet mass flow rate and the maximum wall temperature and maximum oil temperature, the inlet mass flow rate is taken as the abscissa and the ordinate is the temperature, as shown in FIG. 5.

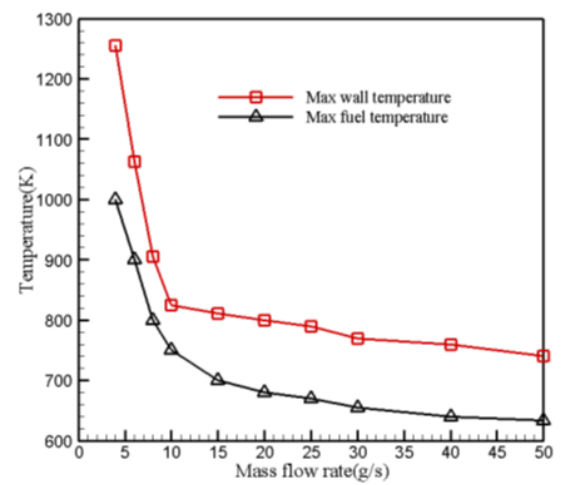

Figure 5. Temperature comparison of different inlet mass flows

It can be found from Figure 5 that the larger the inlet mass flow, the lower the maximum wall temperature and oil temperature. When the inlet mass flow rate is less than $8 \mathrm{~g}$ / s, the maximum wall temperature and oil temperature rise sharply as the mass flow rate decreases. When the inlet mass flow rate is greater than $20 \mathrm{~g} / \mathrm{s}$, the maximum wall temperature and oil temperature decrease as the mass flow rate increases. Slower. Considering that the higher the mass flow rate is, the larger the flow rate is under the condition of constant cross-sectional area. When the flow velocity is too large, the viscosity influence is large, the flow resistance is also large, and the kinetic energy loss of kerosene in the channel is faster. Therefore, the inlet mass flow rate is more suitable between $\quad 8 \mathrm{~g} / \mathrm{s}-20 \mathrm{~g} / \mathrm{s}$.

\subsection{Influence of inlet pressure}

Figures 6 and 7 show the distribution of wall surface temperature and central flow temperature at the centerline of the lower wall surface of the cooling channel with the channel under different inlet pressure conditions. The change trend of wall temperature is basically the same under the three inlet conditions. All of them increase sharply, then stabilize and then abruptly increase, and then decrease near the outlet. The temperature is almost unchanged, and the temperature of the central flow is not much different under different conditions. Consistently showing a linear growth trend, the temperature difference is not greater than $5 \mathrm{~K}$ under different inlet conditions.

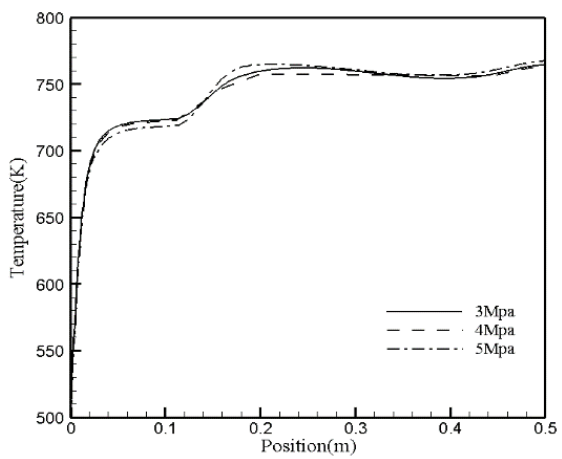

Figure 6. Distribution of temperature at the centerline of wall with channel 


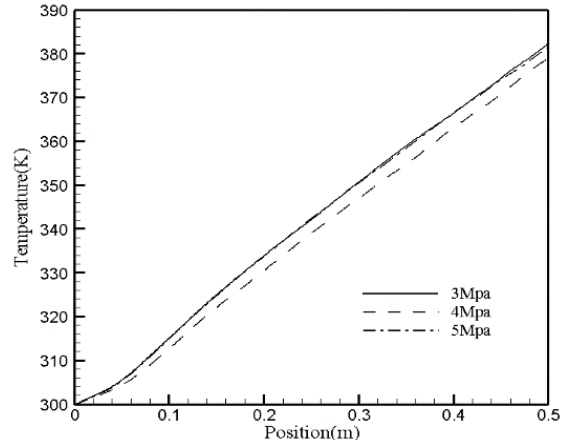

Figure 7. Distribution of central flow temperature with channel.

When the inlet flow rate is $10 \mathrm{~g} / \mathrm{s}$ and the inlet temperature is $300 \mathrm{~K}$, the cases where the inlet pressures are $3 \mathrm{MPa}, 4$ $\mathrm{MPa}$, and $5 \mathrm{MPa}$ are analyzed. Three cross sections in the channel (inlet cross section $\mathrm{z}=0 \mathrm{~m}$, center plane $\mathrm{z}=0.25 \mathrm{~m}$, outlet section $\mathrm{z}=0.5 \mathrm{~m}$ ) was analyzed for temperature distribution, as shown in Figure 8、 Figure 9 and Figure 10.

Comprehensive analysis shows that under different inlet pressures, the temperature difference is small. This is because under the condition of the same inlet mass flow rate and constant inlet temperature, an increase in pressure will cause an increase in kerosene density and dynamic viscosity, resulting in a decrease in flow velocity and Reynolds number. Therefore, pressure has little effect on the temperature of kerosene in the channel.

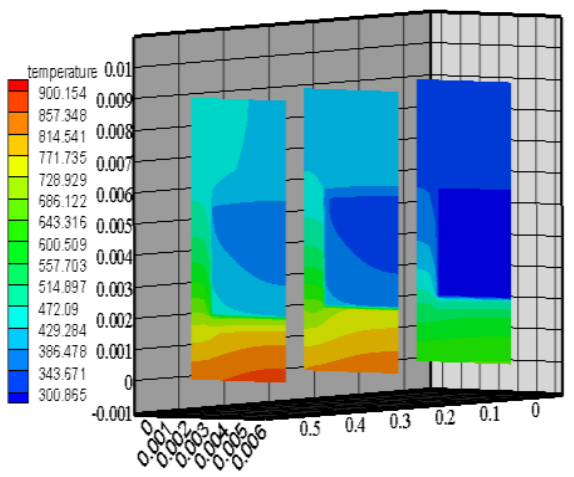

Figure 8. Cloud map of channel temperature distribution at $3 \mathrm{MPa}$.

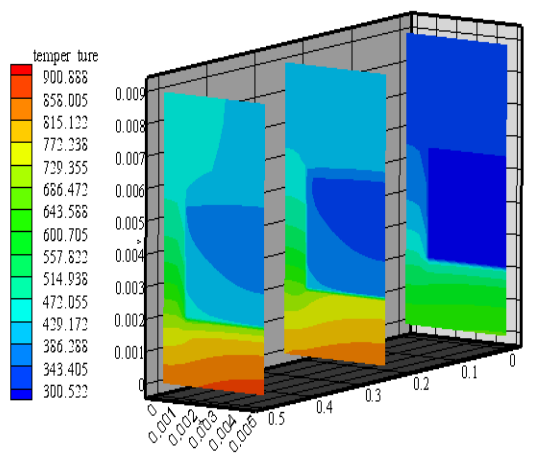

Figure 9. Cloud map of channel temperature distribution at 4MPa.

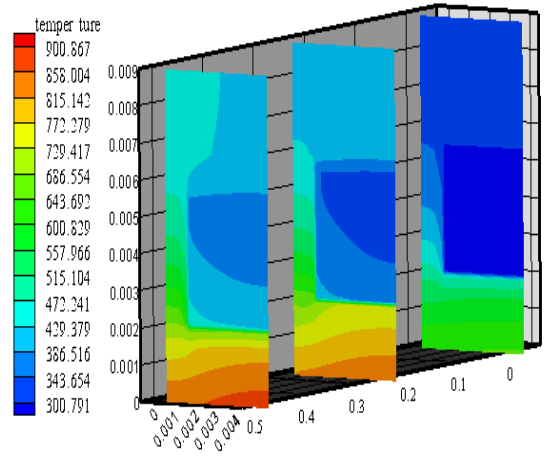

Figure10. Cloud map of channel temperature distribution at $5 \mathrm{MPa}$.

\subsection{Influence of inlet temperature}

In order to study the influence of the inlet temperature on the heat transfer performance of the cooling channel, this section analyzes the cooling channel when the inlet temperature is $200 \mathrm{~K}, 300 \mathrm{~K}, 400 \mathrm{~K}$, and $500 \mathrm{~K}$ when the inlet mass flow is $0.01 \mathrm{~kg} / \mathrm{s}$ and the inlet pressure is $4 \mathrm{MPa}$. Convection heat transfer situation.

Figures 11 to 14 show the distribution of the centerline wall temperature, center flow oil temperature, kerosene pressure, and heat transfer coefficient along the channel at different inlet temperatures. It can be seen from Fig. 11 that when the inlet temperature is $300 \mathrm{~K}, 400 \mathrm{~K}$, and $500 \mathrm{~K}$, the wall temperature of the inner wall of the inner wall varies, and the wall temperature suddenly rises at $0.11 \mathrm{~m}, 0.19 \mathrm{~m}$, and $0.31 \mathrm{~m}$, respectively, and the heat transfer deteriorates. Then it decreases, and the change near the exit section is small. When the inlet temperature is $200 \mathrm{~K}$, the wall temperature does not change abruptly. This is because the temperature of kerosene is low and cannot reach the temperature at which kerosene deteriorates, and no heat transfer deterioration occurs. It can be seen from FIG. 12 that the temperature change trend of the central flow is basically the same, and both gradually increase along the axial direction. The influence of inlet temperature on the pressure of kerosene is mainly reflected at the entrance. As can be seen from Figure 13, when kerosene flows in the channel, the pressure gradually decreases along the channel. The kerosene pressure is almost the same at the temperature of $200 \mathrm{~K}$ and $300 \mathrm{~K}$, but only at the entrance. The kerosene pressure is slightly higher at a temperature of $300 \mathrm{~K}$. When the inlet temperature is high, the corresponding pressure is large, and the pressure loss of kerosene in the channel is also large. When the inlet temperature is low, the pressure loss is small. Figure 14 shows the change of kerosene heat transfer coefficient along the channel. As a whole, the inlet temperature has a greater impact on the heat transfer coefficient. As the inlet temperature decreases, the temperature difference between 
the wall and kerosene becomes larger, and convective heat transfer The coefficient gradually increases. Observe the curves when the inlet temperature is $300 \mathrm{~K}, 400 \mathrm{~K}$, and $500 \mathrm{~K}$. When the wall temperature rises sharply during the inlet stage, the heat transfer coefficient decreases sharply. When the wall temperature does not change much, the heat transfer coefficient also remains almost unchanged. When

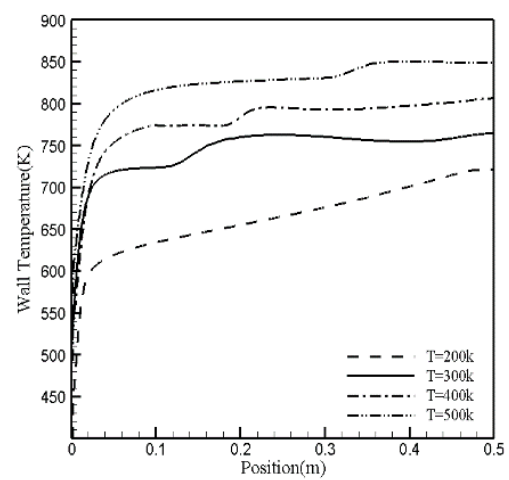

Figure 11. Distribution of wall temperature along the centerline of the inner wall.

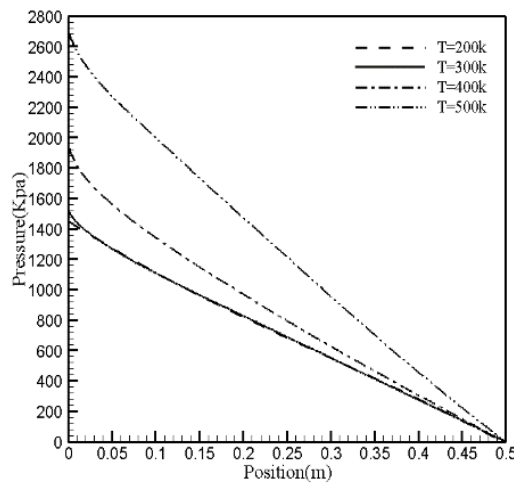

Figure 13. Distribution of central flow kerosene pressure along the channel.

For a more intuitive analysis of the cooling channel and the distribution of kerosene temperature in the channel, Figures 15 to 18 show three cross-sections in the channel (inlet cross-section $\mathrm{z}=0 \mathrm{~m}$, center plane $\mathrm{z}=0.25 \mathrm{~m}$, outlet cross-section $\mathrm{z}=0.5 \mathrm{~m}$ ) at four inlet temperatures. Because the lower wall surface of the cooling channel contacts the engine combustion chamber, the temperature is much higher than the upper wall surface, and the temperature difference is about $400 \mathrm{~K}$. There is a large heat flow near the engine combustion chamber side, which causes a large temperature change. The temperature distribution has a the wall temperature changes suddenly, The heat transfer coefficient decreases again, and finally the wall temperature tends to be constant, and the change of the heat transfer coefficient is relatively small. There is no deterioration of heat transfer on the wall surface at the inlet temperature of $200 \mathrm{~K}$, and the heat transfer coefficient only shows a sharp decline and then a gentle decline.

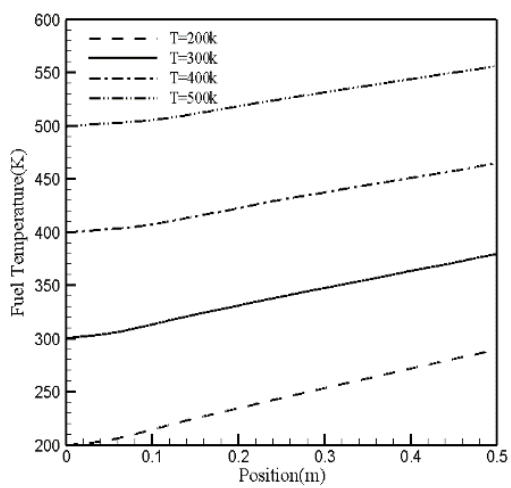

Figure 12. Distribution of central flow oil temperature along the channel.

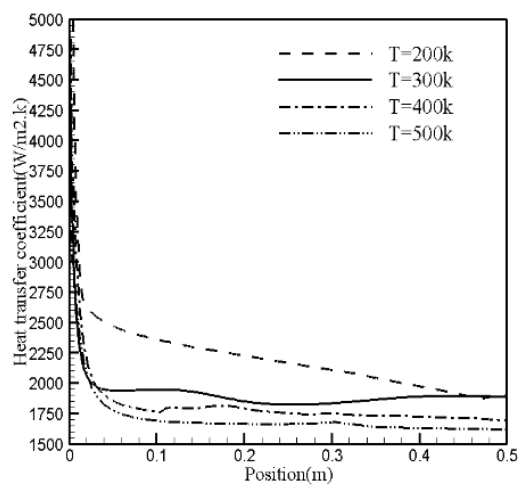

Figure 14. Distribution of heat transfer coefficients along the channel.

more pronounced rib effect. Similarly, kerosene has a large temperature gradient near the lower wall surface and the fin side. With the increase of the inlet temperature, the temperature of the kerosene in the channel increases, the temperature of the lower wall gradually increases, and the temperature of the lower wall changes greatly at different inlet temperatures, while the temperature of the upper wall changes less. The smaller the inlet temperature, the larger the temperature difference between kerosene and the lower wall surface, and the better the heat transfer performance. 


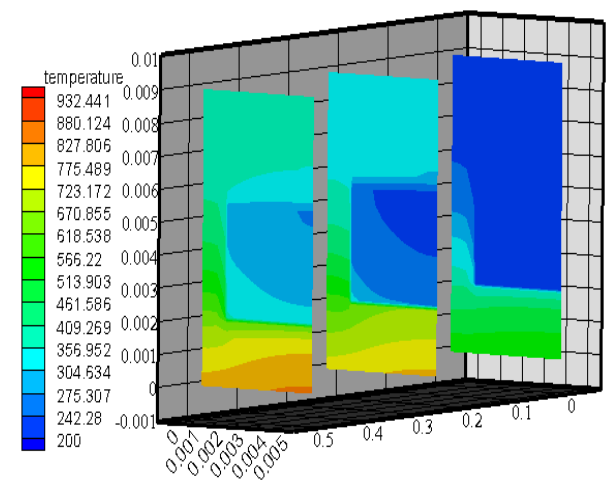

Figure 15. Cloud diagram of channel temperature distribution at inlet temperature of $200 \mathrm{~K}$.

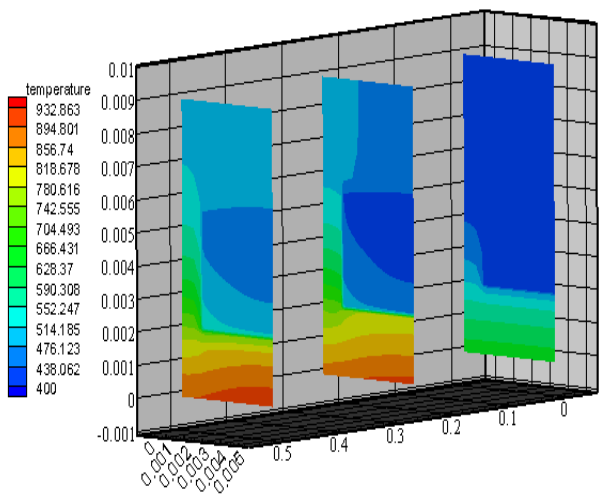

Figure 17. Cloud diagram of channel temperature distribution at inlet temperature of $400 \mathrm{~K}$.

\section{Summary}

This paper proposes a CFD simulation analysis method for regenerative cooling channels, and performs threedimensional modeling and analysis using the current mature fluid mechanics calculation software Fluent. The temperature field of the cooling channel and the temperature distribution of the inner wall surface are given. The effects of inlet mass flow, inlet pressure, and inlet temperature on the flow and heat transfer characteristics of kerosene in the cooling channel are analyzed, and the conclusions are as follows:

(1) As the inlet mass flow increases, the maximum wall temperature and maximum oil temperature are decreasing. The larger the inlet mass flow, the lower the maximum wall temperature and oil temperature. When the inlet mass flow rate is less than $8 \mathrm{~g} / \mathrm{s}$, the maximum wall temperature and oil temperature rise sharply as the mass flow rate decreases; when the inlet mass flow rate is greater than $20 \mathrm{~g}$ / $\mathrm{s}$, the maximum wall temperature and oil temperature decrease as the mass flow rate increases. Slower.

(2) The trend of wall temperature changes under different

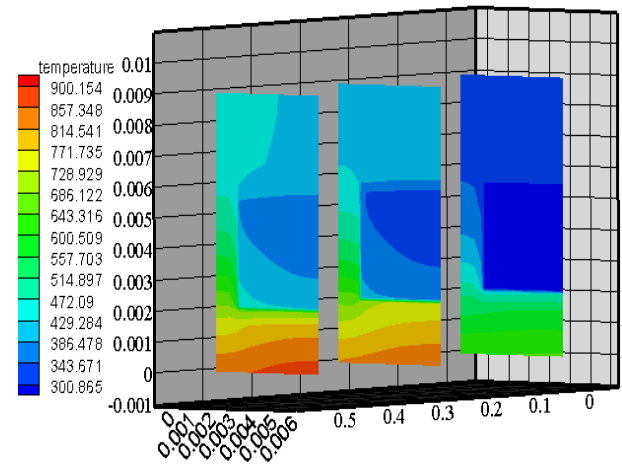

Figure 16. Cloud diagram of channel temperature distribution at inlet temperature of $300 \mathrm{~K}$.

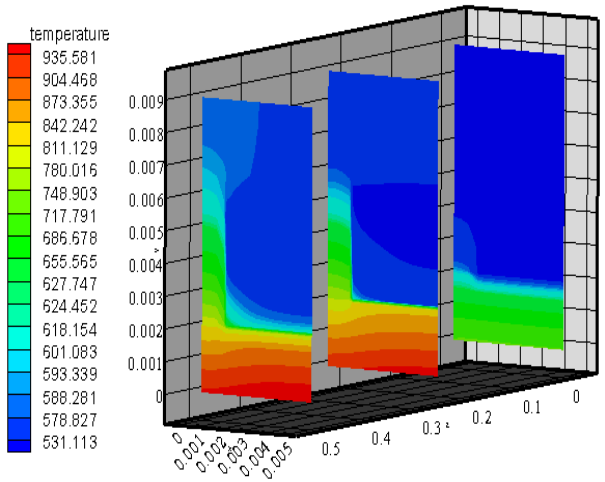

Figure 18. Cloud diagram of channel temperature distribution at inlet temperature of $500 \mathrm{~K}$.

inlet pressure conditions is basically the same, all of which increase sharply, then steadily and then abruptly rise suddenly, and then decrease near the exit, which is almost unchanged, and the temperature difference under different conditions is not large. The temperature of the central flow shows a linear growth trend, and the temperature difference is not greater than $5 \mathrm{~K}$ under different inlet conditions.

(3) The distribution trends of the centerline wall temperature, central flow oil temperature, kerosene pressure, and heat transfer coefficient along the channel are basically the same for different inlet temperatures. When the inlet temperature exceeds $200 \mathrm{~K}$, the centerline wall temperature of the inner wall surface changes abruptly, the wall surface temperature suddenly rises, heat transfer deterioration occurs, and then decreases, and the change near the outlet section is small. When the inlet temperature is high, the corresponding pressure is large, and the pressure loss of kerosene in the channel is also large. When the inlet temperature is low, the pressure loss is small. The inlet temperature has a greater impact on the heat transfer coefficient. As the inlet temperature decreases, the temperature difference between the wall surface and 
kerosene becomes larger, and the convective heat transfer coefficient gradually increases.

\section{References}

1. Xiao Hongyu. Analysis and Design of Reinforced Active Cooling Structure Reinforcement for Supercombustion Stamping Engine [D]. Xi'an: Master's Thesis of Air Force Engineering University, 2007

2. Gong, Les. Real-Time Simulation of Aeroheating of the Hyper-X Airplane [R] NASA. 20110014692, 2011

3. X-51 A scramjet breaks record for longest hypersonic flight [R] .http: // dvicecom / archives / 2010/05 / hypersonic-X-51php, 2010.

4. Ungar, Eugene K .; Schunk, Richard G .. Spacecraft Radiator Freeze Protection Using a Regenerative Heat Exchanger [R] NASA 20110012266,2011

5. Parris, Daniel K .; Landrum, D. Brian. Effect of Tube Geometry on Regenerative Cooling Performance $r$ [R] NASA 20050209950, 2005
6. Stephen J Scotti, Carl J Marthn. Active Cooling Design for Scramjet Engines Using Optimization Methods. AIAA 1988

7. Moholt, Matthew. ARMD Hypersonics Project Materials and Structures: Testing of Scramjet Thermal Protection System Concepts [R]. NASA. 20090012145, 2009

8. A. Frohlich, G. Scheuerer. Three-dimensional flow analysis in a rocket engine coolant channel of hogh depth / width Ratio. AIAA91-2183, 1991

9. Korabelnikov, A. V., Kuranov, A. L .. Thermal protection of hypersonic flight vehicle using chemical heat regeneration [R] .AIAA. A0214063, 2002

10. Leonard CP, Amundsen R M, Bruce III W E. Hyper$\mathrm{X}$ hot structures design and coMParison with flight Data. [R] AIAA 2005-3438, 2005

11. Fan, X J; Yu, G; Li, J G; Lu, X N; Sung. Performance of Supersonic Model Combustors with Distributed Injection of Supercritical Kerosene [R]. AIAA 20075406, 2007 